\title{
LASER SPECTROSCOPY AND AB INITIO CALCULATIONS ON THE TaF MOLECULE
}

KIU FUNG NG, Department of Chemistry, The University of Hong Kong, Hong Kong, Hong Kong; WENLI ZOU, Institute of Modern Physics, Northwest University, Xi'an, China; WENJIAN LIU, Department of Chemistry, Peking University, Beijing, China; ALLAN S.C. CHEUNG, Department of Chemistry, The University of Hong Kong, Hong Kong, Hong Kong.

Electronic transition spectrum of the tantalum monoflouride (TaF) molecule in the spectral region between 448 and $520 \mathrm{~nm}$ has been studied using the technique of laser-ablation/reaction free jet expansion and laser induced fluorescence spectroscopy. TaF molecule was produced by reacting laser-ablated tantalum atoms with sulfur hexafluoride gas seeded in argon. Sixteen vibrational bands with resolved rotational structure have been recorded and analyzed, which were organized into six electronic transition systems and the ground state has been identified to be the $\mathrm{X}^{3} \Sigma^{-}\left(0^{+}\right)$state with bond length, $\mathrm{r}_{o}$, and equilibrium vibrational frequency, $\omega_{e}$, determined to be $1.8209 \AA$ and $700.1 \mathrm{~cm}^{-1}$ respectively. In addition, four vibrational bands belong to another transition system involving lower state with $\Omega=2$ component has also been analyzed. All observed transitions are with $\Delta \Omega=0$. Least-squares fit of the measured line positions yielded molecular constants for the electronic states involved.

The $\Lambda$-S and $\Omega$ states of TaF were calculated at the state-averaged complete active space self-consistent field (SACASSCF) and the subsequent internally contracted multi-reference configuration interaction with singles and doubles and Davidson's cluster correction (MRCISD+Q) levels of theory with the active space of 4 electrons in 6 orbitals, that is, the molecular orbitals corresponding to Ta 5d6s are active. The spin-orbit coupling (SOC) is calculated by the state-interaction approach at the SA-CASSCF level via the relativistic effective core potentials (RECPs) spin-orbit operator, where the diagonal elements of the spin-orbit matrix are replaced by the above MRCISD+Q energies. The spectroscopic properties of the ground and many low-lying electronic states of the TaF molecule will be reported. With respect to the observed electronic states in this work, the calculated results are in good agreement with our experimental determinations. This work represents the first experimental investigation of the molecular structure of the TaF molecule. 